International Conference Mathematical and Computational Biology 2011

International Journal of Modern Physics: Conference Series

Vol. 9 (2012) 278-284

C World Scientific Publishing Company

DOI: $10.1142 / \mathrm{S} 2010194512005326$

\title{
NUMERICAL SOLUTION OF TUMOR-IMMUNE INTERACTION USING 2- POINT BLOCK BACKWARD DIFFERENTIATION METHOD
}

\author{
NOR AIN AZEANY MOHD NASIR \\ Institute for Mathematical Research, Department of Mathematics, \\ Faculty of Science, Universiti Putra Malaysia, \\ 43400 UPM Serdang, Selangor Darul Ehsan, Malaysia \\ ain_nasir2511@yahoo.com.my \\ ZARINA BIBI IBRAHIM \\ Institute for Mathematical Research, Department of Mathematics, \\ Faculty of Science, Universiti Putra Malaysia, \\ 43400 UPM Serdang, Selangor Darul Ehsan, Malaysia \\ zarina@math.upm.edu.my \\ MOHAMED SULEIMAN \\ Institute for Mathematical Research, Department of Mathematics, \\ Faculty of Science, Universiti Putra Malaysia, \\ 43400 UPM Serdang, Selangor Darul Ehsan, Malaysia \\ zarinabb@math.upm.edu.my

\section{K. I. OTHMAN} \\ Department of Mathematics, Faculty of Computer and Mathematical Sciences, \\ Universiti Teknologi MARA, 40450 Shah Alam, \\ Selangor Darul Ehsan, Malaysia \\ khairil@tmsk.uitm.edu.my \\ YONG FAEZAH RAHIM \\ Institute for Mathematical Research, Department of Mathematics, \\ Faculty of Science, Universiti Putra Malaysia, \\ 43400 UPM Serdang, Selangor Darul Ehsan, Malaysia \\ zarinabb@science.upm.edu.my
}

In this paper, we consider tumor-immune interaction model systems. The numerical solutions for the tumor-immune interaction system are obtained by using the 2-point Block Backward Differentiation Formula (BBDF) methods developed by Zarina et al. in 2007. The numerical results are presented in terms of computational time and accuracy of the solutions.

Keywords: Ordinary Differential Equations; Initial Value Problems; Tumor-Immune Interaction. 


\section{Introduction}

In this paper, we focused on solving systems of first order stiff Ordinary Differential Equations (ODEs). The general form for systems of first order stiff ODEs is given as,

$$
Y_{s}^{\prime}=A Y_{s}+F_{s}\left(t, y_{s}(t)\right), \quad Y_{s}(a)=\eta_{s}, \quad s=1,2, \ldots, n .
$$

where $Y_{s}(t)=\left(Y_{1}, Y_{2}, \ldots, Y_{n}\right)^{T}, \quad \eta_{s}=\left(\eta_{1}, \eta_{2}, \ldots, \eta_{n}\right)^{T}, A$ is $m \times m$ matrices with large negative eigenvalues.

Many researchers have proposed numerical methods for solving ODEs such as Chu and Hamilton (1987), Suleiman (1989), Fatunla (1991) and Omar and Suleiman (2003). Although there is extensive literature on using block method for solving Eq. (1), but there are not many works on block methods developed for solving stiff ODEs.

In 2006, Majid and Suleiman (2006) have derived a method which is called 1-point implicit block method of variable stepsize and order (1PVSO) which is presented in the simple form of Adams type. The 1PVSO method is proved to be efficient for solving non-stiff problems within the given tolerance.

Subsequently, Ibrahim et al. (2007) have proposed Block Backward Differentiation Formulas (BBDF) specially for solving stiff ODEs. The BBDF method is constructed in backward difference form to approximate two solutions simultaneously in each block. Since the method is in implicit form, the BBDF method is implemented using Newton's iteration which involved the calculation of the Jacobian and the LU decomposition. This BBDF method has been used for solving the Brusselator by Othman et al. (2008). The results are shown in Othman et al. (2008) to be more efficient when the method is implemented on parallel processors.

Hence, in this paper, we apply the BBDF method for solving tumor-immune interaction model. This mathematical model on tumor-immune interaction is given in Kirschner and Panetta (1998). Most of the tumor-immune interaction equations are stiff ODEs. The aim in this paper is to produce solutions within the user defined tolerance with less computational time. This paper will validate the competency of the BBDF method.

\section{Block Backward Differentiation Formula Method}

In Ibrahim et al. (2007), the derivation of BBDF method are formulated using two backvalues which are $t_{n-1}$ and $t_{n}$. The backvalues are used to compute the approximate solutions concurrently at the points $t_{n+1}$ and $t_{n+2}$. The formulas that have been derived by Ibrahim et al. (2007) are as following,

$$
\begin{aligned}
& y_{n+1}=-\frac{1}{3} y_{n-1}+2 y_{n}-\frac{2}{3} y_{n+2}^{(p)}+2 h f_{n+1}^{(p)} \\
& y_{n+2}=\frac{2}{11} y_{n-1}-\frac{9}{11} y_{n}+\frac{18}{11} y_{n+1}^{(p)}+\frac{6}{11} h f_{n+2}^{(p)}
\end{aligned}
$$


where $p$ is the predicted values. For simplicity, formulas in Eq. (2) can be generally written in the form of,

$$
\sum_{j=0}^{k} A_{j} y_{n+j}=h \sum_{j=0}^{k} B_{j} f_{n+j}
$$

with $A_{j}$ and $B_{j}$ are $r$ by $r$ matrices, where $k$ is the number of blocks and $r$ is the number of points in block.

Equation (2) is then transform into the form of Eq. (3), which is

$$
\left[\begin{array}{cc}
1 & \frac{2}{3} \\
-\frac{18}{11} & 1
\end{array}\right]\left[\begin{array}{l}
y_{n+1} \\
y_{n+2}
\end{array}\right]=\left[\begin{array}{cc}
-\frac{1}{3} & 2 \\
\frac{2}{11} & -\frac{9}{11}
\end{array}\right]\left[\begin{array}{c}
y_{n-1} \\
y_{n}
\end{array}\right]+h\left[\begin{array}{cc}
2 & 0 \\
0 & \frac{6}{11}
\end{array}\right]\left[\begin{array}{l}
f_{n+1} \\
f_{n+2}
\end{array}\right] .
$$

Next, the Eq. (4) is implemented using $P E(C E)^{2}$ mode. $P$ and $C$ indicate one application of the predictor or the corrector respectively, and $E$ indicate one evaluation of the function $f$, given $t$ and $y$. The numerical solution for solving Eq. (1) is obtained as follows

(i) The values of predict solutions are computed using predictor equations,

(ii) Evaluate the values for $e_{n+1, n+2}^{(i+1)}=A^{-1} B$, where $e^{(i+1)}=y_{n+1, n+2}^{(i+1)}-y_{n+1, n+2}^{(i)}$,

$$
\begin{aligned}
& A=\left[\begin{array}{cc}
1-2 h \frac{\partial f_{n+1}}{\partial y_{n+1}} & \frac{2}{3} \\
-\frac{18}{11} & 1-\frac{6}{11} h \frac{\partial f_{n+2}}{\partial y_{n+2}}
\end{array}\right], \\
& B=\left[\begin{array}{cc}
y_{n+1}^{(i)}+\frac{2}{3} h f_{n+1}^{(i)}-\frac{1}{3} y_{n-1}^{(i)}+2 y_{n}^{(i)} & \frac{2}{3} y_{n+2}^{(i)} \\
-\frac{18}{11} y_{n+1}^{(i)} & y_{n+2}^{(i)}+\frac{6}{11} f_{n+2}^{(i)}+\frac{2}{11} y_{n-1}^{(i)}-\frac{9}{11} y_{n}^{(i)}
\end{array}\right]
\end{aligned}
$$

and $\frac{\partial f_{n+1, n+2}}{\partial y_{n+1, n+2}}$ is the Jacobian at ith iteration,

(iii) the values for $y_{n+1, n+2}^{(i+1)}$ is corrected using the values $e_{n+1, n+2}^{(i+1)}$ obtained from (ii),

(iv) the values of $e_{n+1, n+2}^{(i+1)}=A^{-1} B$ is calculated using the LU decomposition and solved for the second stage iteration,

(v) the final values of $y_{n+1, n+2}^{(i+1)}$ is obtained from (iv). 


\section{The Tumor-Immune Interaction Model}

There are many model for tumor-immune dynamic which have been modeled by several researchers such as Kuznetsov et al. (1994), DeLisi and Rescigno (1977), Adam (1996) and DeBoer (1985). However, Kirschner and Panetta (1998) have developed a simple model which consist of the interaction between the effector celss, $E(t)$; the tumor cells, $T(t)$ and the cytokine (IL-2), $I_{L}(t)$. The dynamic model is described as, which is

$$
\begin{aligned}
& \frac{d E}{d t}=c T-\mu_{2} E+\frac{p_{1} E I_{L}}{g_{1}+I_{L}}+s_{1} \\
& \frac{d T}{d t}=r_{2}(T) T-\frac{a E T}{g_{2}+T} \\
& \frac{d I_{L}}{d t}=\frac{p_{2} E T}{g_{3}+T}-\mu_{3} I_{L}+s_{2}
\end{aligned}
$$

with initial conditions $E(0)=E_{0}, \quad T(0)=T_{0}, \quad I_{L}(0)=I_{L_{0}}$. The parameters in Eq. (5) are defined as follows,

Table 1. Parameters definition.

\begin{tabular}{ll}
\hline Parameters & Definition \\
\hline$d E / d t$ & The rate of change for the effector-cell population \\
$d T / d t$ & The rate of change for the tumor cells \\
$d I_{L} / d t$ & The rate of change for concentration of IL-2 \\
$c$ & The measurement of the antigenicity of the tumor \\
$\mu_{2}$ & The natural lifespan of the effector cells \\
$p_{1}$ & The stimulated effector cells by IL-2 per day \\
$g_{1}$ & The concentration of the stimulated effector cells \\
$s_{1}$ & External source of effector cells (treatment) \\
$r_{2}(T)$ & The logistic growth function \\
$a$ & The strength of the immune response \\
$g_{2}$ & The volume loss of the tumor cells \\
$\mu_{3}$ & The degraded rate of IL-2 \\
$s_{2}$ & An external input of IL-2 (treatment) \\
$p_{2}$ & The stimulated effector cells by interaction with the tumor \\
$g_{3}$ & The volume of the stimulated effector cell by interaction with the tumor \\
\hline
\end{tabular}

In Kirschner and Panetta (1998), the parameters values are given below in the table 2. In this work, we only considered $s_{1}$ and $s_{2}$ are equal to zero which means, there is no treatment received by the cancer patient. The value of $c$, the stronger the antigen will produced.

Table 2. Parameters values.

\begin{tabular}{llll}
\hline $0 \leq c \leq 0.05$ & $\mu_{2}=0.03$ & $p_{1}=0.1245$ & $g_{1}=2 \times 10^{7}$ \\
$g_{2}=1 \times 10^{5}$ & $r_{2}=0.18$ & $b=1 \times 10^{-9}$ & $a=1$ \\
$\mu_{3}=10$ & $p_{2}=5$ & $g_{3}=1 \times 10^{3}$ & \\
\hline
\end{tabular}




\section{Numerical Results}

In this section, the numerical results will be tabulated in Table 4 and 5. The BBDF method will be compared with the ode15s and ode23s which runs in Matlab. The notations used in the table are defined as follows,

Table 3. This is the caption for the table. The width of the caption should follow that of the table and centralize if it is less than the table.

\begin{tabular}{ll}
\hline Notation & Definition \\
\hline BBDF & $\begin{array}{l}\text { 2-point Block Backward Differentiation Formula } \\
\text { with fixed stepsize } \\
\text { A variable order solver based on the numerical } \\
\text { ode15s }\end{array}$ \\
& A mofferentiation formulas (NDFs) \\
Ode23s & Method that being compared \\
METHOD & The antigenicity of the tumor \\
$c$ & Stepsize \\
$H$ & Computational time in microseconds \\
TIME &
\end{tabular}

In the literature, the numerical error, Error is defined as the difference between the exact analytical solution $y\left(t_{n+2}\right)$ and its numerical solution $y_{n+2}$, i.e,

$$
\text { Error }=\left|y\left(t_{n+2}\right)-y_{n+2}\right| .
$$

However, in practical situations, most applied problems, the analytic solution is unknown. Hence, one way of computing the error is by using the difference between two approximate values at the end point using different stepsize. Therefore, the numerical error, Error is given as follows,

$$
\text { Error }=\left|y_{n+2}(h)-y_{n+2}(\hat{h})\right| .
$$

where $h=10^{-3}$ and $\hat{h}=10^{-5}$. The error and computational time are presented in Table 4 and 5 .

Table 4. Numerical results for tumor-immune interaction model.

\begin{tabular}{lllll}
\hline$c$ & $H$ & METHOD & Error & TIME \\
\hline \multirow{4}{*}{0.0} & $10^{-3}$ & BBDF & $9.27010 \mathrm{e}-5$ & 7526 \\
& & ode15s & $9.75492 \mathrm{e}-4$ & 125000 \\
& & ode23s & $9.75797 \mathrm{e}-4$ & 140600 \\
& $10^{-4}$ & BBDF & $4.68400 \mathrm{e}-6$ & 48667 \\
& & ode15s & $7.97585 \mathrm{e}-5$ & 218750 \\
& & ode23s & $1.04014 \mathrm{e}-5$ & 187500 \\
& $10^{-5}$ & BBDF & $970000 \mathrm{e}-7$ & 464695 \\
& & ode15s & $5.12100 \mathrm{e}-6$ & 4484375 \\
& ode23s & $2.86021 \mathrm{e}-6$ & 4359400 \\
\hline
\end{tabular}


Table 5. Numerical results for tumor-immune interaction model.

\begin{tabular}{lllll}
\hline$c$ & $H$ & METHOD & Error & TIME \\
\hline \multirow{4}{*}{0.025} & $10^{-3}$ & BBDF & $9.30660 \mathrm{e}-5$ & 7798 \\
& & $9.82605 \mathrm{e}-4$ & 29690 \\
& & ode15s & $9.76929 \mathrm{e}-4$ & 31300 \\
& ode23s & $3.87500 \mathrm{e}-6$ & 49331 \\
& $10^{-4}$ & BBDF & $982372 \mathrm{e}-5$ & 234375 \\
& & ode15s & $8.19604 \mathrm{e}-5$ & 218800 \\
& & ode23s & $977000 \mathrm{e}-7$ & 467225 \\
& $10^{-5}$ & BBDF & $7.75505 \mathrm{e}-6$ & 4046875 \\
& & ode15s & $6.60848 \mathrm{e}-6$ & 5437500 \\
\hline \multirow{4}{*}{$10^{-3}$} & ode23s & $1.01065 \mathrm{e}-4$ & 7438 \\
& & BBDF & $1.01073 \mathrm{e}-3$ & 15625 \\
& & ode15s & $1.12065 \mathrm{e}-3$ & 25000 \\
& & ode23s & $1.48890 \mathrm{e}-5$ & 49016 \\
& & BBDF & $6.32461 \mathrm{e}-4$ & 250000 \\
& & ode15s & $5.11735 \mathrm{e}-4$ & 171875 \\
& & ode23s & $9.85000 \mathrm{e}-7$ & 470719 \\
& & BBDF & $4.22429 \mathrm{e}-6$ & 4656250 \\
& & ode15s & $3.78190 \mathrm{e}-6$ & 4281250 \\
\hline
\end{tabular}

From Table 4 and 5, for all methods, it is obvious that as the stepsize is getting smaller, the error become smaller especially at the last stepsize. The BBDF method outperformed ode15s and ode23s in terms of accuracy. Most of the cases, the error for ode $23 \mathrm{~s}$ is slightly better than the ode $15 \mathrm{~s}$. In terms of computational time, the BBDF method requires less computational time as compared to ode15s and ode23s. On other hand, ode23s produce less execution time compared to ode15s. The BBDF method produced faster solutions because the method is a block method which obtained two solutions values simultaneously at each iteration while ode $23 \mathrm{~s}$ and ode $15 \mathrm{~s}$ is a one point method.

\section{Conclusion}

We have shown the efficiency of the BBDF method in terms of accuracy and computational time over the ode23s and ode15s for solving the tumor-immune interaction model. In conclusion, the BBDF method is a reliable method and can be an alternative solver for solving the tumor-immune interaction problems.

\section{Acknowledgments}

The author gratefully acknowledges the GRF Universiti Putra Malaysia and FRGS for the financial support. 


\section{References}

1. C. DeLisi and A. Rescigno, Immune surveillance and neoplasia I: a minimal mathematical model, in Bull. Math. Biol., 39, 201-221 (1977).

2. D. Kirschner and J. C. Panetta, Modeling immunotherapy of the tumor-immune interaction, in J. Math. Biol., 37, 235-252 (1998).

3. J. A. Adam, Effects of vascularization on lymphocyte/tumor cell dynamics: Qualitative features, in Mathl. Comput. Modelling, 23(6), 1-10 (1996).

4. K. I. Othman, Z. B. Ibrahim and M. B. Suleiman, Parallel block backward differentiation formulas for solving ordinary differential equations, in World Academy of Science, Engineering and Technology, 40, 54-56 (2008).

5. M. B. Suleiman, Solving nonstiff higher order ODEs directly by the direct integration method, in Appl. Math. Comput.., 33(3), 197-219 (1989).

6. M. T. Chu and H. Hamilton, Parallel solution of ODE's by multi-block methods, in Siam J. Sci. Stat. Comput., 8(3), 342-353 (1987).

7. R. J. DeBoer, P. Hogeweg, H. F. J. Dullens, R. A. DeWeger and W. DenOtter, Marcrophage T Lymphocyte interaction in the anti-tumor immune response: A mathematical model, in The Journal of Immunology, 134(4), 2748-2758 (1985).

8. S. O. Fatunla, Block methods for second order odes, in International Journal of Computer Mathematics, 41(1), 55-63 (1991).

9. V. A. Kuznetsov, I. A. Makalkin, M. A. Taylor and A. S. Perelson, Nonlinear dynamics of immunogenic tumors: Parameter estimation and global bifurcation analysis, in Bull. Math. Biol., 56(2), 295-321 (1994).

10. Z. A. Majid and M. B. Suleiman, 1-point implicit code of adams moulton type to solve first order ordinary differential equations, in Chiang Mai J. Sci., 33(2), 153-159 (2006).

11. Z. B. Ibrahim, K. I. Othman and M. B. Suleiman, Implicit $r$-point block backward differentiation formula for solving first-order stiff ODEs, in Applied Mathematics and Computation, 186, 558-565 (2007).

12. Z. Omar and M. B. Suleiman, Parallel r-point implicit block method for solving higher order ordinary differential equations directly,in Journal of ICT, 3(1), 53-66 (2003). 